\title{
Éphémères fiançailles entre l'aménagement du territoire et une vision de développement territorial au Québec ?
}

\author{
Claire Binet
}

On semblait avoir enfin compris récemment au Québec que, pour contrer la poursuite de l'étalement urbain sur le territoire, selon un modèle ruineux au plan des ressources et des valeurs collectives, il ne suffisait pas d'adopter des règles pour encadrer les décisions locales. En effet, l'intérêt public commande d'abord l'énoncé par l'État d'une vision territoriale globale et intégrée ainsi que des interventions cohérentes. Par exemple, on peut imaginer que les villes deviendraient des cités avec une âme, dont on ne chercherait plus à s'enfuir, les campagnes seraient complémentaires aux villes, tout en conservant leurs attributs propres, et les banlieues s'inscriraient en transition ville-campagne plutôt qu'en hybride s'imposant comme modèle spatial.

La matérialisation d'une vision nécessite cependant un mariage à l'échelle humaine de l'aménagement et $\mathrm{du}$ développement territorial ainsi qu'un processus décisionnel offrant formellement à la société civile une voix constructive aux côtés des élus locaux. Mais il semble que, par l'octroi de pouvoirs absolus et largement discrétionnaires aux MRC quant à cette participation de la société civile, le nouveau gouvernement ait choisi d'aller à contre-courant.

\section{Déclin des banlieues à l'horizon et déstructuration rurale}

En ce tournant de siècle, la construction en Occident de grandes régions urbaines, qui concentrent les populations et les activités économiques, est la manifestation actuelle de rapports ville-campagne s'inscrivant dans une trame historique empreinte à la fois d'une domination urbaine et d'une complémentarité ruraleurbaine. Dans un contexte de prévisions démographiques annonçant une relative stagnation et un vieillissement de la population, il faut cependant se préoccuper de la multiplication des banlieues de divers types. Ainsi, doit-on s'attendre à une crise du marché immobilier d'ici une dizaine d'années à cause d'un surplus de résidences unifamiliales ne répondant plus aux nouveaux besoins des ménages? Des signes apparaissent déjà dans les banlieues anciennes où des couples âgées peinent à entretenir des maisons devenues trop grandes. De plus, divers problèmes apparaissent : fermetures d'écoles, besoins de services de santé adaptés à une clientèle vieillissante, difficultés de transport, etc.

Par ailleurs, le redéploiement de populations urbaines dans les zones rurales avoisinantes y cause tout un chambardement. La différentiation entre l'urbain et le rural s'amenuisant constamment, le milieu rural périurbain perd une partie de son essence et de son authenticité, au profit d'une standardisation dominée par des valeurs urbaines. Dans ce contexte, les aspirations des uns et les valeurs des autres ne font pas toujours bon ménage.

\section{La défense de l'intérêt public : coercition ou vision structurante?}

Les moyens employés au Québec depuis 25 ans pour contrôler l'étalement urbain, arbitrer les conflits d'utilisation du sol en milieu périurbain et pour implanter une culture de la planification sur le territoire ont été 
d'une efficacité douteuse ${ }^{1}$. C'est principalement aux instances locales qu'il revient de trancher ces questions et de planifier en fonction de l'intérêt public. Comme les schémas d'aménagement traduisent la vision de municipalités dont la situation financière dépend directement des rentrées de taxes foncières, il est compréhensible que cela influence les orientations retenues.

Jusqu'à maintenant, le rôle des schémas a principalement consisté à établir des balises pour les municipalités composantes. Depuis vingt ans, les orientations d'aménagement du gouvernement québécois ont d'ailleurs davantage ressemblé à un code de conduite pour les municipalités qu'à une incitation à générer des projets rassembleurs. Cet encadrement vise essentiellement à exercer un contrôle, mais les objectifs de bien commun ne sont pas toujours facilement perceptibles. Ce qu'on a demandé aux élus locaux jusqu'à maintenant, c'était d'imposer interdictions, contentions et normes aux divers acteurs du terrain. Bref, un programme bien peu ragoûtant $a$ priori.

\section{Depuis vingt ans, les orientations d'aménagement du gouvernement québécois ont d'ailleurs davantage ressemblé à un code de conduite pour les municipalités qu'à une incitation à générer des projets rassembleurs.}

Par ailleurs, même si les MRC ont vingt ans, les querelles de clocher sont loin d'avoir été oubliées. Les édiles tendent à conclure des alliances entre eux ou à parfois s'abstenir de prendre parti, mais ils renoncent rarement aux aspirations locales, souvent motivées par des intérêts particuliers. Il a sans doute été d'autant plus laborieux de mobiliser les élus municipaux depuis vingt ans autour des exercices d'élaboration des schémas d'aménagement et d'adoption des règlements d'urbanisme qu'aucune vision de développement du territoire n'était proposée. On a mis la charrue avant les bœufs : comment peut-on planifier les vocations d'un territoire si on n'a pas vraiment déterminé les objectifs auxquels ces affectations doivent répondre et encore moins la vision de développement qui doit sous-tendre l'élaboration de ces objectifs?
C'est là que le manque de vision globale et l'absence de projet collectif se font le plus fortement sentir. Si l'on proposait d'abord une vision claire pour un développement global ainsi que des objectifs particuliers permettant d'y parvenir, il serait plus aisé de justifier l'introduction d'obligations, de contraintes et de normes dans l'anticipation de certains résultats positifs pour le bien commun. Jusqu'à récemment, le seul énoncé gouvernemental comportant une vision territoriale était l'Option préférable d'aménagement pour la région de Montréal (1978 et 1983), mais il n'a jamais été mis en œuvre.

\section{L'aménagement au service du développement}

Sous l'angle du développement agroalimentaire, on peut considérer que la Loi sur la protection du territoire agricole de 1978 (LPTA) faisait partie d'une politique d'ensemble axée sur une vision de revitalisation et de diversification de l'agriculture québécoise. D'autres outils de base ont aussi été créés ou remaniés à la même époque (crédit agricole, assurances agricoles, etc.). Pour " nourrir le Québec », comme on le disait alors, il fallait d'abord s'assurer d'une assise territoriale, dans un contexte de rareté de sols arables, de spéculation et d'urbanisation anarchique. À certains égards, la loi a joué son rôle pendant quelques années, quoique parfois un peu brutalement, rappelons-le. Le principal problème avec la LPTA, c'est qu'elle a beaucoup joué un rôle d'aménagement par défaut, ce qui demeure (hélas!) encore trop souvent le cas.

Depuis plusieurs années, les conseils régionaux de développement (CRD) des régions administratives procèdent à l'élaboration de plans stratégiques de développement. Les ententes cadres qui lient les CRD et le gouvernement du Québec ne sont toutefois guère arrimées avec les schémas d'aménagement des MRC et il n'est pas rare d'observer des orientations contradictoires entre ces deux outils d'intervention, surtout dans le domaine agroalimentaire. On parle de concertation depuis tellement d'années, aurait-on raté ce rendez-vous majeur? Il faut dire que l'implication des municipalités locales dans le développement est relativement récente, la culture municipale ayant peu de tradition en cette matière. 
Depuis 2001, le gouvernement a cherché à faciliter une plus grande intégration de l'aménagement et du développement. Dans ses orientations d'aménagement révisées, il a d'abord invité les MRC à s'inspirer des ententes-cadres de développement des CRD pour se doter d'un plan de développement de la zone agricole. Par la suite, la LAU a été discrètement modifiée pour obliger les MRC à « énoncer une vision stratégique de développement culturel, économique, environnemental et social » dans le contenu des schémas afin de faciliter une exercice cohérent de leurs compétences. De plus, les schémas sont dorénavant appelés «schémas d'aménagement et de développement». Enfin, les MRC ont maintenant le pouvoir d'adopter des plans de développement de leur territoire, mais ces plans doivent notamment tenir compte de ceux des CRD et des CLD ${ }^{2}$.

Ces deux mesures ne semblent guère avoir soulevé d'enthousiasme dans les MRC, dont la plupart se sentent lésées par le zonage agricole. Les élus locaux ont généralement préféré déployer leurs énergies à résister au resserrement de l'urbanisation de même qu'à tenter d'obtenir l'exclusion de superficies additionnelles de la zone agricole et un élargissement de leurs pouvoirs pour régir la cohabitation de l'agriculture et des autres activités. Il est clair que ces nouvelles mesures n'ont pas contribué à accélérer le rythme extrêmement lent de la révision des schémas, laquelle est en cours depuis presque dix ans. Entre-temps, l'étalement urbain s'est généralisé sur le territoire, les populations se diffusant de façon croissante en périphérie des agglomérations, ce qui accentue les conflits de voisinage et entrave le développement agricole $^{3}$. Avec une expertise de support aux municipalités qui reste encore teintée d'une vision urbaine, les plans et règlements d'urbanisme découlant des schémas ne sont-ils pas, par leur simple appellation, une invite aux municipalités rurales à copier les villes ?

\section{L'étalement urbain s'est généralisé sur le territoire, les populations se diffusant de façon croissante en périphérie des agglomérations, ce qui accentue les conflits de voisinage et entrave le développement agricole.}

Il est fort regrettable que le grand chantier d'aménagement du territoire des vingt dernières années se soit mis en place sans vision globale de développement territorial pour mettre à contribution l'identité québécoise et sa créativité. Cette grave omission a eu un impact considérable, car on a ainsi laissé toute la place à l'american dream, dont le modèle spatial a fortement imprégné le territoire québécois, surtout pour les villes et leur périphérie rurale. Paradoxalement, pendant que ce modèle américain s'implantait ici en rupture avec l'organisation traditionnelle du territoire, le Québec se démarquait dans les domaines politique, économique, social et culturel par une évolution originale, créative et ancrée dans son identité. Est-il encore possible de ramener le bateau de l'aménagement dans nos eaux?

\section{Des balises pour une vision convergente de développement et d'aménagement ?}

L'élaboration d'une vision d'aménagement et de développement est une responsabilité partagée. D'une part, le gouvernement dispose de pouvoirs et de ressources, mais il a également la responsabilité de proposer une vision globale intégrant l'ensemble des secteurs d'activité et de supporter les municipalités. D'une certaine façon, la Politique nationale de la ruralité annoncée à la fin de 2001 va dans cette direction, par une approche à la fois horizontale (multisectorielle) et territoriale (échelle des MRC). D'autre part, le palier municipal a l'obligation de gérer l'occupation du territoire, en déterminant les différentes vocations, et de fournir un certain nombre de services de proximité à la population, aux entreprises et aux institutions. Les particularités de chaque milieu peuvent cependant influencer la vision locale et la mise en commun de ces deux visions peut nécessiter des ajustements. C'est aussi l'occasion d'arrimer le contenu (la vision de développement) et le contenant (les affectations du territoire).

La récente transition vers des schémas d'aménagement et de développement correspond en quelque sorte à l'introduction d'une troisième génération de schéma qui pourrait permettre d'atteindre une convergence en matière de développement et d'aménagement du territoire. Cela ne peut toutefois se concrétiser qu'avec une proposition gouvernementale d'ensemble pour l'aménagement et le développement du territoire devant servir de référence aux MRC pour définir leurs choix. En fait, le processus a déjà été partiellement amorcé par la publication d'un cadre 
d'aménagement pour les nouvelles communautés métropolitaines créées pour Montréal (CMM) et Québec (CMQ) ${ }^{4}$. Ces deux ensembles urbains coïncident grossièrement avec le territoire des régions métropolitaines de recensement de Montréal et de Québec; ils regroupent environ $60 \%$ de la population totale du Québec.

Dans le Cadre d'aménagement pour Montréal publié en 2001, le gouvernement fait son autocritique et annonce ses couleurs :

Cependant, si l'on en juge par la planification qui a été faite au cours des vingt dernières années, le développement de la région pourrait continuer d'engendrer des coûts économiques, sociaux et environnementaux élevés ainsi qu'un gaspillage de ressources (p. 3).

Ces choix se traduisent notamment par une stratégie générale de gestion de l'urbanisation où s'incarnent ces principes de développement durable. [...] (p. 4).

[...] Le Cadre d'aménagement métropolitain affirme désormais l'importance de concilier l'aménagement et le développement du territoire d'une façon durable et concertée. [...] Le but ultime du Cadre d'aménagement est d'accompagner l'ensemble des acteurs de la région métropolitaine vers un projet rassembleur [...] qui permettra de positionner la région métropolitaine en 2021 parmi les grandes agglomérations de qualité dans le monde (p. 5).

Voulant prêcher par l'exemple, le gouvernement s'est engagé à appliquer ce cadre à l'ensemble des interventions de ses ministères, organismes et mandataires sectoriels, en incluant leurs plans stratégiques. Cette recherche de cohérence et de rationalisation des choix décisionnels pourrait inaugurer une nouvelle ère dans la planification sur le territoire québécois. Si l'on peut se réjouir de cette nouveauté, il faut cependant s'attendre à ce que le chemin soit laborieux; il est peu probable que soit respectée l'échéance de 2006 pour l'adoption, par chacune des deux communautés métropolitaines, d'un schéma métropolitain d'aménagement et de développement. À cet égard, des attentes précises sont exprimées par le gouvernement dans les cadres d'aménagement et divers engagements de soutien sectoriel ont été mis de l'avant.
Par ailleurs, on peut déplorer que cette vision gouvernementale globale intégrant l'aménagement et le développement soit limitée aux deux plus grandes agglomérations du Québec. Peut-on espérer que cette amorce forgera un modèle inspirant pour le reste du territoire? Comment la démarche des conférences régionales d'élus qui seront créées en vertu de la loi 34 récemment adoptée pourrait-elle évoluer dans cette direction? Nous y reviendrons. Au préalable, regardons de plus près la vision proposée pour la CMM et la CMQ.

\section{Une nouvelle vision métropolitaine : solidarité, attractivité, compétitivité, viabilité}

Soulignant l'une des principales conséquences de l'absence de vision qui a prévalu antérieurement, à savoir la dispersion de la population et d'une partie des activités économiques, ainsi que les effets d'entraînement sur la circulation automobile et la congestion routière, le Cadre d'aménagement pour Montréal prend d'emblée parti pour un coup de barre dans l'autre direction. Référant à un modèle que les Américains appellent maintenant TOD (Transit Oriented Development), l'accent est mis sur la densification urbaine et l'utilisation systématique des réseaux de transport en commun. Contrairement au modèle qui a prévalu depuis 40 ans, ce ne sont plus les autoroutes mais les tracés des modes lourds de transport public (métro, train, bus express) qui deviendraient en quelque sorte le squelette de la forme urbaine. Cette vision préconisant un sévère contrôle de l'urbanisation, on peut déjà imaginer les résistances qui vont surgir, et pas seulement dans les chaumières !

\section{Contrairement au modèle qui a prévalu depuis 40 ans, ce ne sont plus les autoroutes mais les tracés des modes lourds de transport public (métro, train, bus express) qui deviendraient en quelque sorte le squelette de la forme urbaine.}

Ce choix s'inscrit dans la foulée du Plan d'action québécois sur les changements climatiques qui évoque les avantages d'un contrôle plus minutieux de l'expansion urbaine et d'une réduction de l'utilisation de l'automobile. La vision proposée mise sur « la diversité biologique et l'utilisation rationnelle des ressources et sur une éthique sociale dans l'utilisation de 
ces ressources, tant entre les générations qu'entre les différents groupes de la société » (p. 64). Chacune des grandes missions de l'État québécois est contenue dans cette vision, dont voici un aperçu :

Mission sociale :

- coût environnemental et social de la croissance payé par ceux qui en profitent;

- quartiers anciens devenus des éléments importants de l'identité régionale : grande qualité de vie urbaine;

- voisinages denses, démarqués par des espaces publics favorisant des échanges civiques.

Mission éducative et culturelle :

- solide réseau d'écoles publiques primaires et secondaires pensé en vue d'enrichir la dynamique locale communautaire;

- ensembles patrimoniaux et attributs culturels bien conservés; dimension culturelle de l'eau et de la nature.

Mission économique et environnementale :

- développement urbain d'abord axé sur l'existant : ère du redéveloppement urbain;

- densification et resserrement des effets de l'expansion urbaine : consolidation des zones urbaines et des pôles économiques existants (forme urbaine polycentrique), tout en préservant boisés et espaces verts et riverains;

- zone agricole composante de l'écosystème urbain, partie intégrante du sentiment d'appartenance régional et apport de l'agriculture au dynamisme économique, revitalisation de terres en friche.

Mission de gouverne et de justice :

- citoyens engagés dans la vie civique et démocratique pour une qualité de vie;

- fort sentiment d'appartenance contribuant au dynamisme de la région.

\section{L'ensemble de cette vision de I'aménagement est-elle utopiste? Pourrait-elle résulter de la maturité des processus de planification au sein du gouvernement du Québec?}

Le concept d'organisation spatiale pour la CMM comporte neuf éléments, à commencer par un centre d'agglomération avec des quartiers centraux, six pôles économiques prioritaires et une couronne périurbaine. Celle-ci côtoie un axe industriel est-ouest, un axe de services nord-sud, la zone agricole ainsi qu'un réseau d'espaces verts et de bassins d'eau. Sur le pourtour extérieur du territoire, on note la présence d'un réseau d'agglomérations moyennes et une couronne à caractère champêtre. Il est important de noter que le cadre d'aménagement s'applique en partie à ce territoire périmétropolitain.

Du côté de Québec, le document de consultation suggère sur plusieurs aspects des orientations similaires à celles proposées pour Montréal (urbanisation et milieux de vie, transport, agriculture, ressources naturelles et environnement). Soulignons notamment que, pour les deux communautés métropolitaines, les orientations préconisées en matière agricole dépassent les préoccupations habituelles de protection du territoire agricole et de cohabitation harmonieuse des activités, pour englober les dimensions paysagère et patrimoniale ainsi que les espaces boisés et humides. Pour la région de Québec, un intérêt particulier est exprimé pour la gestion de l'eau par bassin versant et pour l'intégration d'une agriculture multifonctionnelle dans un cadre urbain et périurbain.

L'ensemble de cette vision de l'aménagement est-elle utopiste ? Pourrait-elle résulter de la maturité des processus de planification au sein du gouvernement du Québec ? Une telle rationalisation pourrait aussi être en grande partie dictée par l'état des finances publiques après des années d'endettement et par une forte pression pour affecter plus de ressources financières dans le réseau de la santé. Mentionnons cependant que les actions antérieures ont été en partie une réponse à une forte et soudaine demande des baby boomers, dans un contexte de croissance économique, avec un appareil d'état jeune et peu expérimenté. Aujourd'hui, la situation diffère : la population augmente peu, elle vieillit et a tendance à se concentrer dans des régions urbanisées. En outre, les ressources financières et naturelles sont fragilisées et de nouvelles valeurs émergent.

\section{Nouvelles valeurs et tendances}

La vision ainsi que plusieurs des orientations de ces cadres d'aménagement métropolitains donnent à prime abord l'impression de faire preuve de beaucoup 
d'audace. En réalité, les valeurs qui les sous-tendent sont déjà présentes dans les débats publics et diverses initiatives depuis plusieurs années; elles rejoignent aussi les préoccupations d'une portion croissante de la population et s'inscrivent dans de nouvelles tendances.

Parmi celles-ci, mentionnons la qualité de vie et les aménités, qui comprennent diverses caractéristiques prisées pour un cadre de vie. Qu'il s'agisse des qualités d'un milieu naturel ou bâti, ou encore de la diversité des activités sportives et culturelles, ces avantages s'ajoutent aux facteurs attractifs habituels d'un lieu. Ils peuvent théoriquement intervenir dans le choix d'un site par de nouveaux résidents ou des entreprises. On peut aussi y relier des besoins sociaux comme la recherche d'une plus grande convivialité pour combattre l'anonymat et un certain isolement vécus en ville ou en banlieue.

Malgré une désaffection de beaucoup de citoyens pour la chose politique depuis quelques années, on note parallèlement un intérêt grandissant pour un engagement dans la communauté. Celui-ci s'appuie sur des sentiments d'identité et d'appartenance locales, lesquels peuvent être alimentés par la connaissance d'un milieu, de son évolution et de ses caractéristiques ${ }^{5}$. À cet effet, les dimensions patrimoniale et culturelle, notamment par la mise en valeur des ressources locales, jouent un rôle catalyseur dans l'enracinement de la population et le ciment qui solidifie une communauté ${ }^{6}$. Enfin, la synergie de tous ces ingrédients est d'autant facilitée par un cadre de mixité sociale et culturelle de même que par un pont intergénérationnel.

Des nouvelles tendances en matière d'urbanisme sont inspirantes ${ }^{7}$. Signalons certaines nouvelles banlieues américaines plus denses, avec des commerces et des services accessibles à distance de marche. Dans certains cas, on a même bâti de toute pièce des rues principales à l'ancienne afin de répondre aux besoins de socialisation et d'identité locale. À Portland (Oregon), on a démoli une autoroute urbaine pour recréer des espaces conviviaux dans des quartiers centraux. Par ailleurs, plusieurs petites villes de la Nouvelle-Angleterre et de l'Ontario ont su préserver leur cachet originel tout en y insérant de nouvelles activités économiques ${ }^{8}$. On peut aussi observer ce phénomène dans plusieurs pays d'Europe comme la

France et l'Allemagne, où un aménagement fonctionnel des lieux reflète les caractéristiques et l'identité des villes et des villages.

\section{Malgré une désaffection de beaucoup de citoyens pour la chose politique depuis quelques années, on note parallèlement un intérêt grandissant pour un engagement dans la communauté.}

\section{Un rôle moteur pour l’agriculture}

$\mathrm{Au}$ sein d'une population essentiellement urbaine à forte origine rurale, comme c'est le cas au Québec, il ne faut pas s'étonner de constater un engouement et une certaine nostalgie pour la campagne ainsi qu'un désir d'espaces ouverts et naturels. Si les activités de plein air sont de plus en plus populaires, il faut aussi compter avec le grand intérêt, voire la passion, que semblent développer les Québécois depuis quelques années pour le jardinage et la cuisine, ce dont témoigne notamment un foisonnement d'émissions de télévision et de publications spécialisées. On peut aussi relier cette tendance à des préoccupations face à la qualité de l'environnement et des aliments qui sont produits et consommés (OGM, traçabilité, etc.).

À cet égard, les espaces agricoles répondent en partie à cette demande pour la campagne et présentent un triple avantage : ces espaces ouverts coûtent moins cher à entretenir que des parcs, ils contribuent au dynamisme économique local et mettent en scène des acteurs du milieu. L'exemple d'une centaine d'hectares en production maraîchère depuis plus de trente ans à Saint-Leu, en banlieue parisienne, représente un cas concret d'intégration réussie ${ }^{9}$. Certains prétendent même que «la meilleure manière de conserver la campagne agricole vivante et dynamique est d'en faire un paysage pour les urbains ${ }^{10}$. Une telle alliance contribue à une mise en valeur agricole dynamique et intégrée à proximité des agglomérations, mais elle peut aussi aider à contrer l'étalement urbain.

Beaucoup de paysages prisés au plan touristique et patrimonial ont été façonnés par des générations d'agriculteurs et de forestiers. C'est notamment la raison 
pour laquelle la préservation et la mise en valeur des paysages est stratégique : "Si la France est, pour les touristes, agréable à regarder et, pour les résidents, douce à habiter, c'est que le spectacle n'a pas souvent été laissé au hasard ${ }^{11}$. Ainsi, les parcs naturels régionaux français maintiennent une agriculture viable sur des territoires préservés pour leurs caractéristiques historiques ou biologiques. Par ailleurs, on a récemment commencé à planter des arbres sur les toits des grands édifices de Chicago alors que, dans plusieurs grandes villes d'Europe et d'Amérique du Sud, on jardine depuis longtemps sur les toits et les terrasses ${ }^{12}$.

Il est nécessaire de responsabiliser et d'encadrer davantage les élus municipaux à l'égard d'une vision de développement qui va au-delà des retombées des infrastructures et de la fourniture de services, qui constituent généralement l'essentiel de l'activité économique et sociale des municipalités.

En outre, « la campagne ne se consomme pas seulement avec les yeux, mais aussi avec une fourchette ${ }^{13}$. De nouvelles formes d'agriculture se sont développées depuis une quinzaine d'années au Québec et ailleurs : produits de niche à valeur ajoutée (biologiques, artisanaux, transformés), autocueillette, vente, visites et repas à la ferme, agriculture à temps partiel, fermes éducatives, etc. Malgré une localisation au cœur de l'agglomération montréalaise et d'importantes pertes de terres agricoles jusqu'en 1990, Laval, troisième ville en importance au Québec, n'en est pas moins devenue sa capitale horticole, avec un fourmillement d'entreprises et d'organismes voués au développement bioalimentaire.

\section{Des retombées attendues}

Peut-on être optimiste en matière de planification de l'aménagement du territoire et de contrôle de l'urbanisation au Québec ? Dans la mesure où l'on prend appui sur une vision de développement territorial ainsi que sur des réalisations concrètes et des exemples réussis, il est permis d'espérer que les choses puissent prendre une tournure plus harmonieuse, après plus de vingt ans de tâtonnements marqués d'erreurs et de maladresses. La concrétisation de cette vision pourrait signifier une capacité d'attraction et de rétention accrue pour des agglomérations régénérées où l'on aurait restauré la mixité des fonctions et des types de résidents, en y prévoyant aussi une répartition des activités et une organisation de l'espace favorisant la convivialité et la sécurité. En outre, la réintroduction en milieu urbain de composantes prisées du milieu rural pourrait aider à refaçonner des villes plus humaines et plus vivables, où une plus grande qualité de vie pourrait détourner les citadins de la tentation de fuir vers la banlieue ou la campagne.

Si de tels résultats n'étaient que partiellement atteints, l'étalement urbain et les pressions sur les terres agricoles diminueraient tout de même significativement. Dans ce nouveau contexte, l'agriculture pourrait être considérée à part entière dans la vie économique et sociale des régions urbaines. En prime, les gestionnaires publics réaliseraient d'importantes économies, notamment grâce à une meilleure gestion de l'urbanisation; ces sommes pourraient alors être réinvesties pour améliorer la qualité de vie de la population et la compétitivité des agglomérations.

Pour ce faire, le gouvernement québécois a le devoir de proposer une vision territoriale globale d'aménagement et de développement ainsi que de favoriser une planification intégrée. À cet égard, les cadres d'aménagement proposés pour Montréal et Québec sont déjà un premier jalon prometteur. Par ailleurs, il est nécessaire de responsabiliser et d'encadrer davantage les élus municipaux à l'égard d'une vision de développement qui va au-delà des retombées des infrastructures et de la fourniture de services, qui constituent généralement l'essentiel de l'activité économique et sociale des municipalités.

Même si les grandes villes tirent de la patte depuis plusieurs années, elles ont néanmoins la capacité de faire preuve de leadership pour entraîner les autres agglomérations dans leur sillage. Il leur revient de se retrousser les manches et de donner le ton pour renverser la vapeur. Certaines initiatives sont à cet effet encourageantes, entre autres la revitalisation du quartier Saint-Roch à Québec et la nouvelle planification stratégique à Lévis. De plus, la confection d'un schéma métropolitain d'aménagement et de développement pour les nouvelles communautés métropolitaines de Montréal et de Québec, lesquelles englobent le territoire de plusieurs MRC, pourrait aider à une meilleure planification d'ensemble. Du 
côté des MRC, la transition vers une troisième génération de schémas, intégrant aménagement et développement, ainsi que l'adoption de plans de développement pourraient donner des résultats similaires.

Il reste beaucoup à faire pour développer une vision territoriale et intégrée, trouver des mécanismes de rééquilibrage fiscal et relancer le chantier de l'aménagement pour que l'ensemble du territoire et des activités y trouve son compte. Le défi est à la fois stimulant et incontournable, car il faut insuffler plus de cohérence, de force et de complémentarité aux différentes entités du territoire québécois, dorénavant en concurrence directe avec d'autres collectivités de la planète.

\section{Des lendemains qui déchantent}

L'aboutissement des longs efforts d'apprentissage de la planification territoriale décrit ci-avant semble toutefois fragilisé, voire compromis, par une récente action du gouvernement élu en avril 2003. En effet, le projet de loi 34, adopté à toute vapeur avant Noël 2003 , vient changer radicalement les règles du jeu. S'il était de toute évidence opportun et nécessaire de donner plus de place aux élus municipaux en matière de développement à l'échelle régionale et locale, il ne l'était certes pas d'écarter de la scène les nombreux organismes de la société civile qui ont vigoureusement contribué au développement des régions depuis une vingtaine d'années. Alors qu'on aurait pu remédier aux lacunes d'imputabilité, il est peu sage d'éliminer une formule gagnante; le fort mouvement d'opposition qui s'est levé spontanément en témoigne.

\section{La récente évolution de la planification de l'aménagement du territoire semblait s'orienter vers un arrimage longtemps déficient entre l'aménagement et le développement du territoire. Dans ce nouveau contexte, il aurait été hautement souhaitable de mettre plus formellement à contribution la société civile pour aider à orienter le travail de planification du monde municipal.}

Comme nous l'avons démontré, la récente évolution de la planification de l'aménagement du territoire semblait s'orienter vers un arrimage longtemps défi- cient entre l'aménagement et le développement du territoire. Dans ce nouveau contexte, il aurait été hautement souhaitable de mettre plus formellement à contribution la société civile pour aider à orienter le travail de planification du monde municipal. Au contraire, l'assujettissement relatif de la société civile au nouveau pouvoir discrétionnaire des $\mathrm{MRC}$ va à contre-courant des nouvelles tendances de gouvernance, alors qu'on observe un engagement croissant des citoyens dans leurs communautés, et risque d'affaiblir le Québec collectivement. De plus, il est inquiétant que ces pouvoirs municipaux additionnels en matière de développement ne soient pas encadrés par une vision gouvernementale adéquate de développement territorial.

\section{On peut s'interroger sur la participation et I'influence réelles qu'aura la société civile quant à cette éventuelle vision des organismes qui remplaceront les CLD et les CRD.}

Cette nouvelle situation aura sans doute des impacts sur l'aménagement du territoire, quoique difficiles à mesurer à cette étape-ci; on peut tenter d'identifier trois avenues de réflexion :

- La vision stratégique de développement visant à faciliter l'exercice cohérent des compétences des MRC requise depuis décembre 2002 dans les schémas d'aménagement et de développement risque fort de devenir en même temps celle des CLD, voire des CRD. On peut s'interroger sur la participation et l'influence réelles qu'aura la société civile quant à cette éventuelle vision des organismes qui remplaceront les CLD et les CRD, comparativement à celles auxquelles elle contribue actuellement au sein des organismes existants.

- L'éventuelle adoption de plans de développement par les MRC en vertu de la LAU perd son sens même, puisque ces plans doivent tenir compte des plans stratégiques des CRD et des plans d'action local des CLD. Or, ce sont les élus municipaux qui décideront partout. Au déficit démocratique s'ajoute donc une concentration des pouvoirs.

- Peut-on s'attendre du côté gouvernemental à la publication prochaine d'une vision globale de déve- 
loppement territorial pour encadrer les orientations des MRC ? À défaut, il est loin d'être sûr que seront maintenues et, surtout, appliquées les orientations des cadres métropolitains d'aménagement publiés pour les communautés métropolitaines de Montréal et Québec. Dans le même ordre d'idées, on ne sait pas ce qu'il adviendra alors des schémas métropolitains d'aménagement et de développement prévus pour ces territoires.

Rappelons que, tout comme pour les récents cadres métropolitains d'aménagement, le contrôle de l'étalement urbain était l'une des principales préoccupations de l'Option préférable d'aménagement pour la région de Montréal de 1978 et 1983 . Or, cet outil gouvernemental de référence avait été ignoré lors de l'approbation des schémas d'aménagement de première génération à partir de 1986. Par ailleurs, le moratoire sur la construction d'autoroutes dans la région métropolitaine de Montréal avait été levé en 1988, en pleine révision de la zone agricole, ce qui avait accentué les pressions sur les terres arables. En outre, la LPTA avait été modifiée en 1989 pour éliminer certains irritants et assouplir son impact.

\section{L'impasse dans laquelle se trouve la révision des schémas d'aménagement depuis quelques années est reliée à la gestion de la zone agricole et à l'étalement urbain}

Signalons ici quelques coïncidences étranges. L'impasse dans laquelle se trouve la révision des schémas d'aménagement depuis quelques années est reliée à la gestion de la zone agricole et à l'étalement urbain, tandis que plusieurs demandes d'exclusion sont en attente de décision à la CPTA. Au cours de l'automne 2003, le gouvernement annonçait le prolongement de l'autoroute 30 sur des terres agricoles à haut potentiel de l'ouest de la Montérégie. Or, ce tracé a été rejeté par la CPTA et le monde agricole s'y s'oppose fortement, à cause des importantes superficies de terres qui seront enclavées dans le voisinage de l'autoroute et exposées à de fortes pressions d'urbanisation. Lors du congrès de l'Union des producteurs agricoles en décembre 2003, la ministre de l'Agriculture avouait publiquement son impuissance : ce dossier avait fait l'objet d'un engagement électoral important de son parti et la décision était déjà prise au soir de l'élection.
Par la même occasion, la ministre a annoncé pour le printemps 2004 des modifications pour « revamper » la LPTA après 25 ans.

Les cadres d'aménagement connaîtront-ils le même sort que l'Option préférable d'aménagement? À quoi peut-on maintenant s'attendre ? Nul ne le sait. Finalement, il se pourrait bien que s'avère fructueuse la stratégie qu'auraient adopté de nombreux élus municipaux à l'effet de chercher à gagner du temps en retardant la révision des schémas d'aménagement, dans l'espoir que le récent changement de gouvernement vienne alléger les règles concernant la zone agricole. Les paris sont maintenant ouverts et avec eux, les inquiétudes grandissent. La vigilance est, dans ce nouveau contexte, plus que jamais de mise.

\section{Notes et références}

1 Voir notre article Le contrôle de l'étalement urbain au Québec : 25 ans de valse-hésitation également publié dans ce numéro.

2 Loi 77 adoptée à l'Assemblée nationale du Québec en décembre 2002 : article 2 modifiant l'article 5 de la LAU, article 3 ajoutant l'article 79.20 à la LAU et article 52 .

3 Binet, Claire (1999). Les espaces agricoles des franges urbaines et le contrôle de l'urbanisation : le cas de la rivesud de Montréal, Montréal, UQAM, rapport d'activité pour la maîtrise en Analyse et gestion urbaines.

4 Voir www.mamsl.gouv.qc.ca: le Cadre d'aménagement pour la Communauté métropolitaine de Montréal (CMM) a été rendu public en juin 2001 alors que celui pour la Communauté métropolitaine de Québec (CMQ) est demeuré au stade de document de consultation, la dernière version remontant à décembre 2002.

5 Le Réseau des Sociétés d'aide au développement des collectivités (SADC) publie annuellement un recueil d'initiatives qui décrit divers projets réalisés au cours de l'année dans les communautés desservies. On y trouve plusieurs exemples d'actions en ce sens.

6 Le programme européen LEADER2 a donné lieu jusqu'en 2001 à de nombreux projets mettant ces éléments à contribution : voir les actions innovantes et les études de cas de coopération transnationale sur le site Internet de l'Observatoire des territoires ruraux : europa.eu.int/comm/archives/leader2

7 Binet, Claire (2000). «Pour un urbanisme renouvelé : la reconquête de la ville par la campagne », conférence prononcée lors d'un déjeuner-conférence de l'Union municipale de Beauport, mars. 
8 Fulford, Robert (2003). « Perceptions of Rural and Urban Ontario Today », conférence prononcée lors de la conférence annuelle du Ontario Rural Council, octobre. (R. Fulford est chroniqueur au National Post.)

9 Fleury, André et Céline Riard. «L'agriculture périurbaine, une nature pour la ville », Métropolis, numéros 101-102.
10 Donadieu, Pierre (1998). «Campagnes urbaines », Versailles, Actes Sud/École nationale supérieure du paysage et Institut national de recherche agronomique (INRA).

11 Idem.

12 Binet, Claire (2000), op. cit.

13 Donadieu, Pierre (1998), op. cit. 